\title{
RADIOTERAPIA E BRAQUITERAPIA NA ENFERMAGEM: UMA REVISÃO BIBLIOMÉTRICA
}

Luciana Martins da Rosa', Marciele Misiak², Monique Mendes Marinho³, Patrícia Ilha ${ }^{4}$, Vera Radünz', Vivian Costa Fermo ${ }^{5}$

${ }^{1}$ Enfermeira. Doutora em Enfermagem. Professora da Universidade Federal de Santa Catarina. Florianópolis-SC-Brasil.

2Enfermeira. Mestre em Enfermagem. Professora do Instituto Federal de Santa Catarina. Florianópolis-SC-Brasil

${ }^{3}$ Enfermeira. Mestre em Enfermagem. Hospital Universitário da Universidade Federal de Santa Catarina. Florianópolis-SC-Brasil.

${ }^{4}$ Enfermeira. Mestranda em Enfermagem. Universidade Federal de Santa Catarina. Florianópolis-SC-Brasil.

${ }^{5}$ Enfermeira. Mestre em Enfermagem. Florianópolis-SC-Brasil.

RESUMO: Estudo bibliométrico e cienciométrico que identificou e caracterizou as publicações da enfermagem referentes à radioterapia e braquiterapia no âmbito nacional. A coleta de dados foi realizada em agosto de 2013, com busca nas Bases de Dados da Enfermagem, Literatura Latino-Americana em Ciências de Saúde, Medical Literature Analysis and Retrieval System Online e Scientific Electronic Library Online através dos descritores "enfermagem", "radioterapia" e "braquiterapia". Incluídas 30 publicações, analisadas por estatística descritiva simples cujos resultados mostraram que $43,33 \%$ dos conhecimentos foram produzidos por mestres e mestrandos, a maioria vinculada às instituições de ensino da Região Sudeste; $56,66 \%$ publicadas em periódicos classificados com estratos A1 e B1; $20 \%$ abrangeram a prevenção, identificação e tratamento de complicações e efeitos colaterais; os estudos foram predominantemente qualitativos. A produção de conhecimento na área investigada é incipiente, por isso a necessidade de maior investigação a fim de melhorar o cuidado às pessoas submetidas às essas modalidades terapêuticas. DESCRITORES: Enfermagem oncológica; Oncologia; Cuidados de enfermagem; Pesquisa em enfermagem; Bibliometria.

\section{RADIOTHERAPY AND BRACHYTHERAPY IN NURSING: A BIBLIOMETRICAL REVIEW}

\begin{abstract}
Bibliometric and scientometric study that identified and characterized the nursing publications regarding radiotherapy and brachytherapy in Brazil. Data collection was performed in August 2013, with search in the Nursing Database, Latin American Literature in Health Sciences, Medical Literature Analysis and Retrieval System Online and Scientific Electronic Library Online, using the descriptors "nursing", "radiotherapy" and "brachytherapy". It included 30 publications analyzed by simple descriptive statistics and the results showed that $43.33 \%$ of knowledge was produced by M.Sc. graduates and students, mostly linked to educational institutions in the Southeast; $56.66 \%$ published in journals classified as strata A1 and B1; $20 \%$ covered prevention, identification and treatment of complications and side effects; the studies were predominantly qualitative. The production of knowledge in the investigated area is incipient; therefore, there is the need for more research to improve the care to people subject to these treatment modalities.
\end{abstract}

DESCRIPTORS: Oncologic nursing; Oncology; Nursing care; Nursing research; Bibliometrics.

\section{RADIOTERAPIA Y BRAQUITERAPIA EN LA ENFERMERÍA: UNA REVISIÓN BIBLIOMÉTRICA}

RESUMEN: Estudio bibliométrico y cienciométrico que identificó y caracterizó las publicaciones de enfermería acerca de la radioterapia y braquiterapia en el ámbito nacional. Los datos fueron obtenidos en agosto de 2013, con búsqueda en las Bases de Datos de la Enfermería, Literatura Latinoamericana en Ciencias de Salud, Medical Literature Analysis and Retrieval System Online y Scientific Electronic Library Online a través de los descriptores "enfermería", "radioterapia" y "braquiterapia". Hubo 30 publicaciones, analizadas por estadística descriptiva simple cuyos resultados mostraron que $43,33 \%$ de los conocimientos fueron producidos por maestros y maestrandos, la mayoría de las instituciones de enseñanza de la Región Sudeste; $56,66 \%$ publicadas en periódicos clasificados con A1 e B1; $20 \%$ abordaron prevención, identificación y tratamiento de complicaciones y efectos colaterales; predominaron los estudios cualitativos. La producción de conocimiento en el área investigada es incipiente, por eso hay necesidad de más investigaciones para mejorar el cuidado a las personas sometidas a esas modalidades terapéuticas. DESCRIPTORES: Enfermería oncológica; Oncología; Cuidados de enfermería; Investigación en enfermería; Bibliometría.

Universidade Federal de Santa Catarina

Rua Cabreuvas, 88 - 88137-435 - Palhoça-SC-Brasil

E-mail: moniquemarinho@yahoo.com.br 


\section{INTRODUÇÃO}

Estimativas mundiais da Organização Mundial de Saúde (OMS) apontaram 14,1 milhões de casos novos de câncer e um total de 8,2 milhões de mortes pela doença em 2012. Para o ano de 2030 está prevista a ocorrência de 21,4 milhões de casos novos e 13,2 milhões de mortes por câncer no mundo. Em países em desenvolvimento, o aumento de sua incidência é decorrente do crescimento e envelhecimento da população, redução da mortalidade infantil e das mortes por doenças infectocontagiosas. A estimativa para os anos de 2014 e 2015 no Brasil mostra a ocorrência de aproximadamente 576 mil casos novos de câncer, revelando a magnitude e relevância de tratarmos essa questão como um problema de saúde pública que deve ser trabalhado(1).

O câncer, por estar na maioria das vezes associado ao sofrimento e dor no imaginário popular, gera ao paciente grande instabilidade emocional durante a vivência da doença. Somase ainda o fato do tratamento exigir períodos de internação, procedimentos e tecnologias invasivas que aumentam a imunossupressão e acarretam graves efeitos colaterais. Assim, a enfermagem, por atuar diretamente no cuidado ao paciente oncológico, requer habilidades emocionais e conhecimento técnico e científico a fim de oferecer cuidado de qualidade e seguro ao paciente e seus familiares, comtemplando os aspectos biopsicossociais ${ }^{(2)}$.

$\mathrm{Na}$ busca por assistência adequada e resolutiva, o desenvolvimento tecnológico permitiu aprimorar as técnicas bem como ampliar as formas de tratamento oncológico. A radioterapia é uma das principais modalidades terapêuticas, e consiste na aplicação de radiação ionizante produzida por aparelhos ou emitida por radioisótopos naturais, aplicada diretamente no local, à distância (teleterapia) ou junto ao tumor (braquiterapia) onde foi identificada a neoplasia. Essa aplicação na maior parte das vezes é realizada em regime ambulatorial ${ }^{(3)}$.

As inovações tecnológicas exigem dos enfermeiros reformulação nas formas de pensar, ser e agir diante das exigências e requisitos da prática assistencial e de ensino. Torna-se necessário o investimento em pesquisas que focalizem as situações do cotidiano profissional e que produzam conhecimentos válidos para melhoria do cuidado nos distintos cenários de atuação ${ }^{(4)}$. Assim, levantou-se o seguinte questionamento: Quais são as principais características das publicações da enfermagem, no âmbito nacional, referentes à radioterapia e a braquiterapia? A partir desta questão norteadora traçou-se o objetivo de identificar as publicações da enfermagem, no âmbito nacional, referentes à radioterapia e à braquiterapia e suas principais características.

\section{METODOLOGIA}

A fim de alcançar o objetivo proposto, optou-se por uso da Pesquisa Bibliométrica e a Cienciometria. A primeira tem como objeto de estudo os livros ou as revistas científicas, cujas análises se vinculam à gestão de bibliotecas e bases de dados. A segunda se preocupa com a dinâmica da ciência, como atividade social, tendo como objetos de análise a produção, a circulação e o consumo da produção científica. Ambas quantificam, constatam e atribuem sentido aos dados, qualificando-os para que possam ter melhor uso em políticas de ciência e tecnologia, conhecimentos aplicados às áreas de ciências humanas e de saúde, ampliando a visão dentro de determinadas especialidades ou grupos de pesquisa, ou até mesmo em contextos mais abrangentes, regionais, nacionais ou mundiais ${ }^{(5-7)}$.

Para a construção desta revisão foram percorridas seis etapas: seleção dos termos; definição das bases de dados para busca; estabelecimento dos critérios para seleção da amostra; análise geral dos resultados da busca; construção e preenchimento de um formulário para registro dos dados coletados; análise dos dados e interpretação dos resultados.

As fontes de busca das publicações para realização da pesquisa foram: Literatura LatinoAmericana em Ciências de Saúde (LILACS); Base de Dados da Enfermagem (BDENF); Scientific Electronic Library Online (SciELO); e a Medical Literature Analysis and Retrieval System Online (MEDLINE). Para busca das publicações utilizouse os termos: Radioterapia $A N D$ Enfermagem e Braquiterapia $A N D$ Enfermagem A partir desses cruzamentos aplicaram-se os seguintes critérios de inclusão: produção científica no idioma português a fim de buscar a literatura produzida no país, disponível em formato de artigo na íntegra 
em meio eletrônico, e que abordaram de alguma forma o tema "Enfermagem em radioterapia e/ou braquiterapia". Não se aplicou limite de tempo de publicação, a fim de caracterizar toda a produção presente nas bases a respeito do tema. A busca foi realizada em agosto de 2013.

Após o cruzamento dos termos Radioterapia $A N D$ Enfermagem e Braquiterapia $A N D$ Enfermagem foram encontrados 2161 e 220 artigos, respectivamente. Com a implementação dos critérios de inclusão foram selecionados 45 artigos com os descritores Radioterapia AND Enfermagem e 05 artigos com os descritores Braquiterapia AND Enfermagem (Tabela 1 e 2).

Os cinco artigos selecionados para leitura do texto completo através o cruzamento Braquiterapia AND Enfermagem também foram encontrados no cruzamento Radioterapia AND Enfermagem, assim foi realizada a leitura de 45 textos completos. Após, foram excluídos 15 artigos, pois não abordavam o tema em questão, assim, a amostra final foi composta por 30 artigos. As publicações foram analisadas por meio dos dados registrados em formulário produzido por autores do presente estudo, como também anotados em planilhas construídas no Programa Excel da Microsoft. O tratamento dos dados foi por estatística descritiva simples (frequência absoluta e relativa) e apresentados no formato de tabelas e descrição analítica.

Identificou-se nas publicações a procedência, o tipo de estudo, o Qualis $\mathrm{CAPES}^{\left({ }^{(8)}\right.}$ da revista escolhida para publicação, o nível de hierarquia da evidência científica ${ }^{(9)}$ através da análise da metodologia, a titulação e a instituição que o autor principal estava vinculado, e o ano de publicação dos artigos.

Tabela 1 - Etapas de seleção de artigos com os termos Radioterapia AND Enfermagem. Florianópolis-SCBrasil, 2013

\begin{tabular}{|c|c|c|c|c|c|c|c|c|c|c|}
\hline \multirow{3}{*}{ Bases de dados } & \multirow{2}{*}{\multicolumn{2}{|c|}{$\begin{array}{l}\text { Trabalhos } \\
\text { encontrados }\end{array}$}} & \multicolumn{6}{|c|}{ Excluídos/Critérios de exclusão } & \multirow{2}{*}{\multicolumn{2}{|c|}{$\begin{array}{l}\text { Selecionados } \\
\text { para leitura do } \\
\text { texto na íntegra }\end{array}$}} \\
\hline & & & \multicolumn{2}{|c|}{ Outros idiomas } & \multicolumn{2}{|c|}{$\begin{array}{c}\text { Não disponíveis } \\
\text { na íntegra }\end{array}$} & \multicolumn{2}{|c|}{ Repetidos } & & \\
\hline & $\mathbf{n}$ & $\%$ & $\mathbf{n}$ & $\%$ & $\mathbf{n}$ & $\%$ & $\mathbf{n}$ & $\%$ & $\mathbf{n}$ & $\%$ \\
\hline BDENF & 38 & 1,85 & 0 & 0 & 19 & 35,29 & 0 & 0 & 19 & 45,83 \\
\hline LILACS & 78 & 3,61 & 19 & 0,94 & 27 & 52,95 & 16 & 51,61 & 16 & 33,33 \\
\hline SciELO & 22 & 1,02 & 0 & 0 & 0 & 0 & 12 & 38,71 & 10 & 20,84 \\
\hline$\overline{M E D L I N E}$ & 2023 & 93,52 & 2014 & 99,06 & 6 & 11,76 & 3 & 9,68 & 0 & 0 \\
\hline Total & 2161 & 100 & 2033 & 100 & 52 & 100 & 31 & 100 & 45 & 100 \\
\hline
\end{tabular}

Tabela 2 - Etapas de seleção de artigos com os termos Braquiterapia AND Enfermagem. Florianópolis-SCBrasil, 2013

\begin{tabular}{|c|c|c|c|c|c|c|c|c|c|c|}
\hline \multirow{3}{*}{ Bases de dados } & \multirow{2}{*}{\multicolumn{2}{|c|}{$\begin{array}{c}\text { Trabalhos } \\
\text { encontrados }\end{array}$}} & \multicolumn{6}{|c|}{ Excluídos/Critérios de exclusão } & \multirow{2}{*}{\multicolumn{2}{|c|}{$\begin{array}{c}\text { Selecionados } \\
\text { para leitura do } \\
\text { texto na íntegra }\end{array}$}} \\
\hline & & & \multicolumn{2}{|c|}{ Outros idiomas } & \multicolumn{2}{|c|}{$\begin{array}{c}\text { Não disponíveis } \\
\text { na íntegra }\end{array}$} & \multicolumn{2}{|c|}{ Repetidos } & & \\
\hline & $\mathbf{n}$ & $\%$ & $\mathbf{n}$ & $\%$ & $\mathbf{n}$ & $\%$ & $\mathbf{n}$ & $\%$ & $\mathbf{n}$ & $\%$ \\
\hline BDENF & 4 & 1,81 & 0 & 0 & 3 & 25 & 0 & 0 & 1 & 20 \\
\hline LILACS & 10 & 4,54 & 2 & 0,99 & 6 & 50 & 0 & 0 & 2 & 40 \\
\hline SciELO & 01 & 0,46 & 0 & 0 & 0 & 0 & 0 & 0 & 1 & 20 \\
\hline MEDLINE & 205 & 93,19 & 201 & 99,01 & 3 & 25 & 0 & 0 & 1 & 20 \\
\hline Total & 220 & 100 & 203 & 100 & 12 & 100 & 0 & 0 & 5 & 100 \\
\hline
\end{tabular}




\section{RESULTADOS}

A partir da análise dos 30 artigos selecionados para o estudo, foi realizado o levantamento de variáveis que delineavam as características das produções.

O vínculo institucional do autor principal se apresentou com 16 instituições distintas, um estudo não apresentou vínculo institucional. Em relação a esta variável, a Escola de Enfermagem de Ribeirão Preto - Universidade de São PauloUSP se destacou com sete publicações (23,33\%), seguida por Instituto Nacional de Câncer (INCA) e a Universidade Federal de Pelotas na área de enfermagem, ambas responsáveis por quatro estudos (13,33\%). A Escola de Enfermagem da Universidade de São Paulo publicou dois artigos (6,67\%). As demais instituições, Escola de Enfermagem Anna Nery/Universidade Federal do Rio de Janeiro, Universidade Federal de São Paulo, Universidade Federal de São Carlos no estado de São Paulo, Universidade Federal do Rio de Janeiro, Universidade Castelo Branco no estado do Rio de Janeiro, Secretaria Municipal de Aracaju no estado do Sergipe - Centro de Atenção Psicossocial, Universidade Estadual de Campinas, Centro de Radioterapia do Hospital Alemão Oswaldo Cruz no estado de São Paulo,
Universidade Estadual de Maringá, Universidade Estadual Paulista, Universidade Federal do Piauí e Universidade Federal do Rio Grande-FURG, foram, cada uma, responsáveis por um artigo (3,33\%). As instituições responsáveis por um artigo estavam vinculadas à área da Enfermagem, sendo que a grande maioria dos autores possuía vínculo com programas de pós-graduação e os demais estavam ligados às áreas assistenciais da enfermagem.

Em relação à titulação do primeiro autor, a maioria dos artigos foi produzida por mestrandos, totalizando sete publicações (23,33\%), seguida por mestres, com seis publicações (20\%), doutores, especialistas e graduandos, ambos foram autores de três publicações (10\% respectivamente), doutorandos produziram duas publicações $(6,66 \%)$ e a titulação residente produziu uma publicação (3,33\%). Cinco autores não informaram a titulação.

Referente à região da produção do conhecimento destacou-se a região sudeste com 20 artigos (66,66\%), seguida por região Sul com sete artigos (23,33\%). A região Nordeste produziu dois artigos (6,66\%). Em uma produção não foi identificada a informação.

Para a publicação dos estudos foram utilizados 13 periódicos, conforme a Tabela 3.

Tabela 3 - Periódicos com publicações da enfermagem brasileira referentes à radioterapia e braquiterapia. Florianópolis-SC-Brasil, 2013

\begin{tabular}{lcc}
\hline Periódico & Número de publicações & $\%$ \\
\hline Revista Latino-americana de Enfermagem & 9 & 30 \\
\hline Revista Enfermagem UERJ & 5 & 16,67 \\
\hline Revista Brasileira de Cancerologia & 4 & 13,33 \\
\hline Enfermería Global & 2 & 6,68 \\
\hline Revista Brasileira de Enfermagem & 2 & 6,68 \\
\hline Einstein & 1 & 3,33 \\
\hline Revista Eletrônica de Enfermagem & 1 & 3,33 \\
\hline Acta Paulista de Enfermagem & 1 & 3,33 \\
\hline Escola Anna Nery Revista de Enfermagem & 1 & 3,33 \\
\hline Revista Texto e Contexto & 1 & 3,33 \\
\hline Revista Gaúcha de Enfermagem & 1 & 3,33 \\
\hline Revista Escola Enfermagem USP & 1 & 3,33 \\
\hline Ciência, cuidado e saúde & 1 & 3,33 \\
\hline Total & 30 & 100 \\
\hline
\end{tabular}


Dos periódicos selecionados, nove (30\%) pertencem a Classificação Qualis CAPES A1, cinco a $(16,67 \%)$ A2, oito a $(26,67 \%)$ B1, e quatro $(13,33 \%)$ a B2 e B3, respectivamente.

Em relação ao nível de hierarquia da evidência científica, não há estudos de nível I (revisão sistemática de ensaio controlado randomizado e revisão sistemática de ensaios não randomizados), nível II (ensaio controlado individual e ensaio não randomizado) e nível III (revisão sistemática de estudos de correlação/observação). Três artigos (10\%) foram classificados como nível IV (estudo de correlação/observação), cinco artigos (16\%) como nível $V$ (revisão sistemática de estudos descritivos/qualitativos/fisiológicos), 21 artigos (70\%) como nível VI (estudo descritivo/ qualitativo/fisiológico individual) e um artigo (4\%) como nível VII (opiniões de autoridades, comitê de especialistas).

As produções foram publicadas entre o período de 1996 e 2012 (Tabela 4).

Os temas investigados foram reunidos em 10 grupos a partir do objetivo dos estudos, apresentados de forma decrescente, conforme interesse de investigação (Tabela 5).

Quanto ao tipo de estudo, 19 produções (63,33\%) possuíam delineamento qualitativo, 10 quantitativo (33,33\%), e uma publicação (3,33\%) utilizou delineamento misto.
Tabela 4 - Ano de publicação dos artigos. FlorianópolisSC-Brasil, 2013

\begin{tabular}{lcc}
\hline Ano da publicação & $\mathbf{N}$ & $\mathbf{\%}$ \\
\hline 1996 & 1 & 3,33 \\
\hline 2002 & 1 & 3,33 \\
\hline 2003 & 3 & 10 \\
\hline 2004 & 0 & - \\
\hline 2005 & 1 & 3,33 \\
\hline 2006 & 1 & 3,33 \\
\hline 2007 & 2 & 6,67 \\
\hline 2008 & 6 & 20 \\
\hline 2009 & 7 & 23,34 \\
\hline 2010 & 2 & 6,67 \\
\hline 2011 & 3 & 10 \\
\hline 2012 & 3 & 10 \\
\hline Total & 30 & 100 \\
\hline
\end{tabular}

Tabela 5 - Temas de investigação. Florianópolis-SC-Brasil, 2013

\begin{tabular}{lcc}
\hline Tema de investigação & Número de publicações & \% \\
\hline $\begin{array}{l}\text { Prevenção, identificação e tratamento de } \\
\text { complicações/efeitos colaterais }\end{array}$ & 8 & 26,67 \\
\hline Experiências/vivências do paciente com câncer & 6 & 20 \\
\hline O vivenciar do câncer por paciente e seu familiar & 4 & 13,33 \\
\hline A prática assistencial da enfermagem & 3 & 10 \\
\hline $\begin{array}{l}\text { A qualidade de vida dos pacientes submetidos a } \\
\text { radioterapia/braquiterapia }\end{array}$ & 3 & 10 \\
\hline $\begin{array}{l}\text { As repercussões psicológicas relacionadas com o } \\
\text { tratamento por radioterapia/braquiterapia }\end{array}$ & 2 & 6,68 \\
\hline $\begin{array}{l}\text { A consulta de enfermagem ao paciente submetido a } \\
\text { radioterapia }\end{array}$ & 1 & 3,33 \\
\hline $\begin{array}{l}\text { As necessidades de cuidados ao paciente submetido a } \\
\text { radioterapia }\end{array}$ & 1 & 3,33 \\
\hline Perfil epidemiológico & 1 & 3,33 \\
\hline Terapias alternativas & 1 & 3,33 \\
\hline Total & 30 & 100 \\
\hline
\end{tabular}




\section{DISCUSSÃO}

A partir da década de 70 ocorreu uma expansão gradativa dos cursos de pós-graduação em enfermagem no Brasil. Em 1974, o país possuía apenas dois cursos de mestrado, já em 1998 passou a ter 14 cursos de pós-graduação em enfermagem. Em 2009 esse número aumentou para 41 programas de pós-graduação. Em 2014, a pós-graduação em enfermagem apresentou 96 cursos de pós-graduação, sendo 49 mestrados acadêmicos, 32 doutorados e 15 mestrados profissionais $^{(10-11)}$.

O presente estudo mostra que o maior percentual de titulação do primeiro autor é de mestrandos, seguido por mestres e após ocorre uma equivalência entre doutores, graduandos e especialistas. Essa realidade revela que a soma de alunos de pós-graduação strictu senso (mestres e doutores) ultrapassa $50 \%$ dos autores, o que pode ser explicado pela realidade dos programas de pós-graduação apresentar entre suas exigências a produção científica. A divulgação da produção do conhecimento é fator essencial para o ingresso na maioria dos programas de pós-graduação.

Analisando a distribuição dos artigos publicados por regiões brasileiras, foi possível identificar a discrepância significativa do número de produções por estado. Como analisado anteriormente, a produção do conhecimento esta intimamente associada aos cursos de pósgraduação, por isso, é um elemento relevante relacionado à predominância da produção na Região Sudeste, que possui a maior concentração de instituições de ensino superior do país ${ }^{(12-13)}$, como também grande número de empresas nacionais e multinacionais que são grandes financiadoras de programas de pesquisa ${ }^{(14)}$. As instituições de ensino superior atraem investimentos públicos e privados para realização de projetos de pesquisas com o objetivo de produzir novas tecnologias. A quantidade e a qualidade dos produtos finais construídos e disseminados na comunidade científica possibilitam maiores incentivos para novos projetos, o que gera um ciclo de produção da pesquisa e do conhecimento.

Nos estudos realizados, os autores optaram principalmente pelo método qualitativo. Este método é amplamente utilizado nas pesquisas que trabalham com a ciência humana, com a finalidade de entender o significado individual ou coletivo de um determinado fenômeno ${ }^{(15)}$. $\mathrm{Na}$ enfermagem ele é utilizado em pesquisas com objetivo de retratar determinada realidade, por meio de histórias e narrativas, além de experiências, percepções e crenças. Acreditase que a enfermagem, por ter o cuidado como foco, tem grande preocupação com as questões subjetivas que envolvem os seres humanos, a fim de interpretar a realidade do ser cuidado, alcançar a empatia e beneficiar a sua prática profissional. Através da pesquisa qualitativa, os profissionais da enfermagem podem compreender, descrever e explicar como o ser humano vivencia o câncer, seu tratamento e efeitos colaterais, e todos os outros fenômenos complexos que o paciente oncológico e seu familiar podem vir a passar durante o enfrentamento da patologia.

A pesquisa qualitativa aponta um olhar diferenciado para a prática da enfermagem, pois expande as perspectivas ao considerar a complexidade e diversidade do ser. Através da percepção da subjetividade do outro, consegue compreender os fenômenos de interesse para a enfermagem que ajudarão na ampliação e construção do conhecimento, assim como fortalecerão o seu papel social ${ }^{(16)}$.

Como exemplo de publicação qualitativa que compõe a amostra deste estudo, cita-se estudo que realizou abordagem a seis mulheres com câncer de mama que estavam em tratamento radioterápico e as suas percepções acerca da família e das relações familiares ante o contexto do câncer. No discurso dos sujeitos compreendem-se o impacto do diagnóstico para a família e como esta pode ser fonte de apoio e cuidado para o familiar enfermo. Os resultados contribuíram para salientar que a família deve ser um aliado no cuidado ao paciente com câncer, oferecendo apoio, cuidado e força. Por isso, uma das ações dos enfermeiros ao cuidarem da mulher acometida por câncer de mama, é prestar cuidado também ao familiar, pois também se encontram em situação de doença, além de capacitá-los para o cuidado ${ }^{(17)}$. É através de pesquisas qualitativas que podemos identificar aspectos dificilmente visualizados durante o cotidiano profissional e compreender os sentimentos permeados por aqueles que vivenciam o processo saúde-doença.

Por outro lado, os estudos quantitativos são importantes por permitirem estabelecer a 
correlação entre variáveis para ocorrência de determinado fenômeno ${ }^{(18)}$. Por exemplo, uma das publicações da amostra, diz respeito a um estudo prospectivo com 86 mulheres portadoras de câncer de mama, operadas em estadios iniciais, submetidas a radioterapia adjuvante na Universidade Federal de São Paulo e no Hospital Alemão Oswaldo Cruz. A radioterapia foi realizada em região da mama, campos tangentes e paralelos opostos, dose total de 5.040 cGy (180 cGy dose/dia), com acelerador linear de $6 \mathrm{MV}$. Além de coletado os dados pessoais, foi medido o tamanho da mama, a partir de seu desenho do contorno. Mediram-se a distância entre a separação dos campos e a altura da mama. A avaliação da pele da área de tratamento foi realizada semanalmente e as reações foram classificadas a partir do Radiation Therapy Oncology Group (RTOG). O estudo demostrou que a chance de ocorrer reação de pele grau 3 aumenta 2,61 vezes a cada aumento de uma unidade da altura $(\mathrm{cm})$ da mama. Este estudo foi importante, pois demonstrou que quanto maior a altura da mama, maiores são as chances de ocorrer reação de pele grau $3^{(19)}$, e a partir deste resultado, os enfermeiros podem estudar e programar cuidados para otimizar o tratamento e diminuir os efeitos colaterais da radioterapia no tratamento do câncer de mama.

Atualmente, teóricos que estudam o fenômeno do desenvolvimento e da difusão de ideias, elencam a necessidade da prática baseada em evidências como essencial para melhoria nos tratamentos de saúde, sendo que as melhores evidências científicas originam-se de descobertas de pesquisas rigorosas e metodologicamente apropriadas à questão proposta. No topo da hierarquia de evidências que classificam as fontes de dados de acordo com a solidez das informações fornecidas consta a revisão sistemática de ensaios controlados randomizados e revisão sistemática de ensaios não randomizados, seguida por ensaio controlado randomizado individual e ensaio não randomizado (nível II) e revisão sistemática de estudos de correlação/observação (nível III) ${ }^{(18)}$, não presentes na amostra deste estudo. Comprovase a incipiência da produção da enfermagem no âmbito da radioterapia e braquiterapia, sendo necessários maiores investimentos no desenvolvimento de pesquisas e formação de pesquisadores, para que no futuro a produção da enfermagem construa melhores evidências científicas para basear sua prática profissional.

Vale ressaltar que em relação à Classificação Qualis CAPES Periódicos ${ }^{(8)}$, sistema brasileiro de avaliação de periódicos, destacaram-se maior número de publicações em periódicos classificados como estrato A1 e B1. Como os estratos são divididos em oito níveis em ordem decrescente de qualidade (A1, A2, B1, B2, B3, B4, B5 e C), os resultados aqui encontrados demonstram a qualidade das produções sobre essa perspectiva.

Dentre as produções, observou-se a predominância de estudos com a preocupação de contribuir no cuidado de enfermagem ao paciente submetido à radioterapia, como prevenção, identificação e tratamento de complicações e efeitos colaterais, e as experiências e vivências do paciente e seu familiar frente ao diagnóstico e tratamento do câncer. Estes temas têm inquietado os enfermeiros durante seu cotidiano profissional, por isso devem ser estimulados a ficar atentos e sensíveis durante o atendimento ao paciente, a fim de basear questionamentos sobre sua prática profissionais que os levem ao desejo de produzir novos conhecimentos que contribuam para mudanças organizacionais e disseminação de novas tecnologias.

Como ocorreu uma evolução significativa das produções a partir do ano de 2008, com expressivo aumento em 2000, em decorrência da expansão dos cursos de pós-graduação no Brasil, deve-se, como demonstrado em pelo Instituto Nacional do Câncer, melhorar a qualificação dos profissionais da saúde para a área da oncologia através da educação em seus serviços de origem e de ofertas de programas de mestrado e doutorado ${ }^{(20)}$, a fim de favorecer a pesquisa, a produção e o consumo do conhecimento científico, assim como o aumento e o impacto das publicações na prática assistencial da enfermagem.

\section{CONSIDERAÇÕES FINAIS}

Os artigos científicos dos enfermeiros brasileiros, na área da oncologia em radioterapia e braquiterapia, embora tenham crescido nos últimos anos, demonstram que a pesquisa ainda não está consolidada, devido lacunas na produção do conhecimento comprovadas neste estudo. É necessário maior disponibilidade de dados de 
pesquisas de alta qualidade, com a inclusão de ensaios clínicos. Para isso são necessários maiores investimentos na formação de pesquisadores em instituições de ensino e de assistência, com foco no questionamento de melhorias para prática assistencial da enfermagem, busca de acesso às informações e análise crítica da produção científica disponível em periódicos, construção de novos conhecimentos e habilidades para divulgar as tecnologias descobertas.

A pesquisa na enfermagem oncológica brasileira tem por compromisso fornecer conhecimentos que devem promover a qualificação dos cuidados, o aprimoramento das técnicas assistenciais, o desenvolvimento de novas tecnologias, a avaliação dos serviços prestados, a formação dos profissionais, no intuído de prestar cuidado cada vez mais eficaz e seguro ao paciente.

Como limitação deste estudo, apontase a não realização de busca pela literatura internacional, a qual pode trazer contribuições para o desenvolvimento de novas estratégias tanto de cuidado, quanto de pesquisas na área. Sugere-se a construção de estudo que compare a produção nacional com a construída no âmbito internacional.

\section{REFERÊNCIA}

1. Ministério da Saúde (BR). Instituto Nacional do Câncer. Estimativa 2014: Incidência do câncer no Brasil. [Internet] Rio de Janeiro; 2014. Disponível: http://www. inca.gov.br/estimativa/2014/

2. Salimena AMO, Teixeira SR, Amorim TV, Paiva ACPC, Melo MCSCM. O vivido dos enfermeiros no cuidado ao paciente oncológico. Cogitare enferm. 2013;18(1):142-7.

3. Leite FMC; Ferreira FM; Cruz MSA; Lima EFA; Primo CC. Diagnósticos de enfermagem relacionados aos efeitos adversos da radioterapia. Rev. Min. Enferm. 2013;17(4):940-5.

4. Moreira MC, Carvalho V, Silva MM, Sanhudo NF, Filgueira MB. Produção de conhecimento na enfermagem em oncologia: contribuição da escola de enfermagem Anna Nery. Esc. Anna Nery. 2010;14(3):575-584.

5. Santos RNM; Kobashi NY. Bibliometria, Cientometria, Infometria: conceitos e aplicações. Pesq. bras. Ci. Inf. 2009;2(1):155-172.

6. Vanti NAP. Da bibliometria à webometria: uma exploração conceitual dos mecanismos utilizados para medir o registro da informação e a difusão do conhecimento. Ci. Inf. [Internet] 2002;31(2):152-62 [acesso em 10 abr 2014]. Disponível: http://dx.doi. org/10.1590/S0100-19652002000200016

7. Araújo CA. Bibliometria: evolução histórica e questões atuais. Em Questão. [Internet] 2006;12(1):11-32 [acesso em 10 abr 2014]. Disponível: http://revistas. univerciencia.org/index.php/revistaemquestao/article/ viewFile/3707/3495

8. Ministério da Educação (BR). Sistema Integrado Capes. WebQualis [Internet]. Brasília: Ministério da Educação; 2014 [acesso 10 mar 2014]. Disponível: http://qualis.capes.gov.br/webqualis/principal.seam

9. Melnyk BM, Fineout-Overholt E. Making the case for evidence-based practice. In: Melnyk BM, FineoutOverholt E. Evidence-based practice in nursing \& healthcare: a guide to best practice. Philadelphia: Lippincott Williams \& Wilkins; 2005. p. 3-24.

10. Erdmann AL, Fernandes JD, Teixeira GA. Panorama da educação em enfermagem no Brasil: graduação e pós-graduação. Enferm. Foco. 2011;2(suppl):89-93

11. Ministério da Educação (BR). Coordenação de Aperfeiçoamento de Pessoal de Nível Superior [Internet]. Brasília: Coordenação de Aperfeiçoamento de Pessoal de Nível Superior; [acesso em 30 mar 2014]. Mestrados e Doutorados reconhecidos na área de enfermagem; [1 tela]. Disponível: http://conteudoweb. capes.gov.br/conteudoweb/

12. Ministério da Educação (BR). Coordenação de Aperfeiçoamento de Pessoal de Nível Superior. Documento da Área de Enfermagem. 2010. [acesso em 10 mar 2014]. Disponível: http://www. capes.gov.br/images/stories/download/avaliacao/ ENFERMAGEM_22jun10b.pdf.

13. Ministério da Educação (BR). Coordenação de Aperfeiçoamento de Pessoal de Nível Superior. Diretoria de Avaliação. Relatório de divulgação dos resultados finais da avaliação trienal 2010. [acesso em 22 mar 2014]. Disponível: trienal.capes.gov.br/ wp-content/uploads/2011/08/relatorio_geral_dos_ resultados_finais_da-avaliacao_2010.pdf

14. Ministério da Ciência e Tecnologia (BR). Termos de Compromisso de Gestão -TCG - Análise dos resultados obtidos [Internet] 2007 [acesso em 30 mar 2014]. Disponível: http://www.gespublica. gov.br/biblioteca/pasta.2010-12-08.3617032145/ pasta. $2010-04-08.8586550461 /$ pasta.2010-04-08.5823886016/MCT.pdf

15. Turato ER. Métodos qualitativos e quantitativos na área da saúde: definições, diferenças e seus objetivos de pesquisa. Rev Saúde Pública. [Internet] 2005;39(3) [acesso em 10 mar 2014]. Disponível: http://dx.doi. 
org/10.1590/S0034-89102005000300025

16. Lacerda MR, Labronici LM. Papel social e paradigmas da pesquisa qualitativa de enfermagem. Rev. Bras. Enferm. 2011;64(2):359-364.

17. Feijó AM, Schwartz E, Jardim VMR, Linck CL, Zillmer JGV, Lange C. O papel da família sob a ótica da mulher acometida por câncer de mama. Cienc. cuid. saude. 2009;8(Supll):79-84.

18. Polit DF, Beck CT. Essentials of nursing research: appraising evidence for nursing practice. 7th ed. Philadelphia: Lippincott Williams \& Wilkins; 2010. Finding and Reviewing Research Evidence in the Literature. p. 54-82.

19. Pires AMT, Segreto RA, Segreto HRC. Avaliação das reações agudas da pele e seus fatores de risco em pacientes com câncer de mama submetidas à radioterapia. Rev Latino-Am. Enfermagem. 2008;16(5):844-9.

20. Thuler LCS, Bergmann A, Ferreira SC. Ensino em Atenção Oncológica no Brasil: Carências e Oportunidades. Rev. Bras. Cancerol. 2011;57(4):467472. 